Cahiers $d u$ MONDE RUSSE

\section{Cahiers du monde russe}

Russie - Empire russe - Union soviétique et États indépendants

$46 / 4 \mid 2005$

L'invention d'une politique humanitaire

\title{
Lada Vladimirovna Silina, Nastroenija sovetskogo studenčestva, 1945-1964
}

\section{Bella Ostromoukhov}

\section{OpenEdition \\ Journals}

Édition électronique

URL : https://journals.openedition.org/monderusse/6637

DOI : 10.4000/monderusse.6637

ISSN : $1777-5388$

\section{Éditeur}

Éditions de l'EHESS

\section{Édition imprimée}

Date de publication : 1 décembre 2005

Pagination : 935-938

ISBN : 2-7132-2057-2

ISSN : $1252-6576$

Référence électronique

Bella Ostromoukhov, «Lada Vladimirovna Silina, Nastroenija sovetskogo studenčestva, 1945-1964 », Cahiers du monde russe [En ligne], 46/4 | 2005, mis en ligne le 29 juin 2009, consulté le 03 septembre 2022. URL : http://journals.openedition.org/monderusse/6637 ; DOI : https://doi.org/10.4000/ monderusse.6637

Ce document a été généré automatiquement le 3 septembre 2022

Tous droits réservés 


\title{
Lada Vladimirovna Silina, Nastroenija sovetskogo studenčestva, 1945-1964
}

\author{
Bella Ostromoukhov
}

\section{RÉFÉRENCE}

Lada Vladimirovna SILINA, Nastroenija sovetskogo studenčestva, 1945-1964 [L'état

d'esprit des étudiants soviétiques, 1945-1964]. Moscou : Russkij Mir, 2004, 233 p.

1 Le titre de l'ouvrage semble l'inscrire d'emblée dans le courant de l'historiographie actuelle, et ce pour plusieurs raisons. Axé sur un groupe de population défini et facile à cerner, ce travail fait partie des études sur le quotidien, sur le vécu d'individus ordinaires, qui permettent de compléter les travaux d'histoire politique. Cette démarche implique également une ouverture de l'histoire à des méthodes empruntées à d'autres sciences sociales.

2 Le terme d'« état d'esprit " va également dans ce sens. En effet, pour plusieurs historiens russes d'aujourd'hui, il s'agit d'une composante du quotidien'. Cette expression, floue, renvoie à la fois à l'opinion publique, à des prises de position déclarées vis-à-vis d'événements politiques, à des rumeurs diffuses et inclut parfois des formes d'expression non verbales, comme des comportements sciemment déviants. La diversité des phénomènes qu'englobe ce concept en fait précisément un instrument adéquat pour analyser le quotidien. Encore faut-il en définir d'emblée les limites, la signification et la portée. Il s'agirait, selon L. N. Silina qui ouvre le livre par cette définition, de «l'état spirituel de la société, ses aspirations et ses penchants, ses nécessités et ses besoins ».

Enfin la période traitée, qui regroupe l'après-guerre et l'époque Hruščev, laisse à penser que l'auteur s'inscrit dans l'historiographie actuelle qui récuse une compartimentation faisant de la mort de Stalin une fracture absolue. 
4 L'horizon d'attente est ainsi posé : il s'agit d'une période "charnière ", au cours de laquelle «se produit une réévaluation massive des valeurs» (p.3) et l'ouvrage va s'efforcer de saisir l'insaisissable, l'état d'esprit d'un groupe, conditionné, selon Silina, par la réalité sociale et politique, et qui l'influence également en retour.

Peut-on à la lecture du livre estimer cette tentative réussie?

6 Le plan de l'ouvrage n'est pas exempt d'une certaine rigidité, imputable aux exigences académiques du mémoire de thèse dont cet ouvrage est issu. L'auteur commence par décrire ses sources et place son travail dans un triple contexte bibliographique, passant en revue tout d'abord les écrits consacrés aux étudiants en tant que groupe social, puis les ouvrages analysant l'opinion publique en général et les modalités de son expression en URSS en particulier, et enfin les travaux d'historiens concernant l'après-guerre, la guerre froide et la période de la dissidence.

7 Ce préalable est suivi d'un chapitre introductif consacré aux « étudiants soviétiques en tant que phénomène social ». L'auteur tente de saisir la spécificité du milieu social étudié en se fondant sur des données statistiques publiées en URSS en 1971 et en 1977. Il en ressort que c'est un groupe qui s'accroît de manière sensible après la guerre et dont la composition est hétérogène, tant du point de vue de l'âge et de l'origine sociale ou géographique que du vécu préalable (la différence entre ceux qui commencent leurs études supérieures tout de suite après l'école secondaire et ceux qui ont fait la guerre entre ces deux étapes est soulignée à plusieurs reprises). L'autre spécificité de ce groupe social, d'après l'auteur, est son interaction avec les instances d'encadrement comme le Komsomol et le PCUS.

Les deux chapitres suivants décrivent les « humeurs» (traduction littérale de nastroenija) des étudiants à proprement parler et sont structurés en fonction des événements politiques. Le chapitre III est en effet consacré aux premières années de l'après-guerre et englobe en réalité la période stalinienne, tandis que le quatrième concerne la période 1953-1964, marquée par l'ascension puis la chute de Hruščev. L'organisation de ces sections suit le même fil conducteur, ce dont témoignent les sous-titres ${ }^{2}$. On peut donc reprocher à l'ouvrage de construire une réflexion sur le social à partir d'une chronologie d'histoire politique. On se prive par là d'une périodisation alternative, qui serait fondée sur ces mêmes phénomènes sociaux, mais en décalage par rapport aux décisions qui visent à les réguler. D'ailleurs, ce cadrage influe sur la conception même de l'objet d'étude. Qui nierait que la politique de l'État a exercé une influence non négligeable sur les diverses composantes de la société soviétique ? Cependant, réduire l'analyse de cette dernière à l'étude de ses réactions face à la politique revient à lui dénier une dynamique propre et à négliger des facteurs non politiques. L'objet étudié en sort appauvri.

Notre remarque est confortée par la manière dont le groupe analysé est présenté dans l'ouvrage. Une fois défini comme « la partie la plus intellectuelle [de la jeunesse] [...], ceux qui détiennent dans leurs mains l'avenir du pays» (p.4), celui-ci ne semble plus poser de problèmes de définition à l'auteur. Or n'observe-t-on pas dans certains chapitres un amalgame entre ceux qui se désignent, ou sont désignés, comme " jeunes », comme « Komsomols» ou comme « étudiants»? Par exemple, au chapitre III.7, consacré aux réactions des étudiants face à la mort de Stalin, la source principale est constituée par des périodiques "pour jeunes » (p. 87) - essentiellement Komsomol'skaja pravda-, où s'expriment à la fois étudiants, écoliers et jeunes travailleurs (p. 96-97), et dont la parole est citée au même niveau, comme celle des « étudiants ». Certains événements, comme le $\mathrm{VI}^{\mathrm{e}}$ Festival international des étudiants et de la jeunesse (p. 121), ou les récitals de poésie, 
place Majakovskij (p. 123), sont cités comme des manifestations estudiantines, sans que la question de la composition réelle de l'assistance soit posée. De manière plus générale, les opinions du groupe étudié sont souvent présentées comme reflétant celles de la population dans son ensemble. Le lecteur en est amené à se demander ce que c'est qu'être étudiant et ce que cela implique en termes de modes et d'espaces d'expression. Mais à ces questions, l'ouvrage ne fournit que des éléments de réponse sporadiques.

S'interrogeant peu sur la spécificité du groupe étudié, l'auteur n'exploite pas les possibilités que lui offrirait cette différenciation. En effet, certains clivages du milieu étudiant ne sont que brièvement évoqués dans la deuxième partie. On pourrait se demander, par exemple, de quelle manière le type d'établissement (école d'ingénieurs, institut pédagogique, université à vocation généraliste), ou le régime des études (temps plein, cours du soir, externat) influe sur les prises de position des étudiants, ou sur ce qui permet la formation de groupes informels générateurs d'opinions au sein des établissements. À l'inverse, l'ouvrage se cantonne dans une démarche descriptive qui, si elle a l'avantage de présenter en détail certaines formations, ne permet cependant pas d'établir un lien entre les éléments cités. Ainsi, les sociétés secrètes d'étudiants sont énumérées aussi bien dans la partie consacrée aux années 1945 que pour la période Hruščev, sans qu'aucun parallèle soit tracé entre tous ces groupes. Il en résulte une vision fragmentaire du temps et de l'espace. Ces divers groupes se connaissaient-ils entre eux ? Avaient-ils entendu parler les uns des autres? Prenaient-ils position les uns par rapport aux autres? En les décrivant uniquement dans leur rapport d'opposition au pouvoir, l'auteur perd de vue de possibles convergences à l'intérieur du groupe étudié.

11 Ce questionnement insuffisant quant à l'objet d'étude va de pair avec un autre écueil essentiel, à savoir l'absence de recul par rapport aux catégories d'analyse utilisées par les instances encadrantes. L'exemple le plus flagrant concerne la déviance. Alors que les sources auraient pu permettre d'étudier la façon dont les normes comportementales sont définies et négociées entre étudiants et institutions, l'auteur se contente de citer - en guise de preuve d'existence de comportements déviants - les documents qui signalent, par exemple, "des cas fréquents de beuveries, des comportements amoraux, des occurrences d'infraction au régime d'études, le mépris du collectif, le nihilisme affiché dans l'approche de la réalité soviétique " (p.110). Les termes indigènes sont ensuite repris tels quels dans l'analyse, assortis d'explications d'ordre psychologique ou de jugements de valeur. C'est le cas, par exemple, de l' «alcoolisme» signalé par le Comité central du Komsomol, et qui est ensuite évoqué comme un fait avéré, sans que les frontières floues et la subjectivité de ce concept soient évoquées: "L'alcoolisme croissant parmi les jeunes, y compris parmi les étudiants, pendant les premières années de l'après-guerre, a une explication logique: [l'amertume des pertes, les dures épreuves psychiques et physiques] [...]. Cependant, certains anciens combattants ont bien trouvé des forces pour commencer une vie nouvelle et surmonter leurs peines » (p. 49).

Pour conclure, le livre dresse un vaste panorama des comportements et des prises d'opinion d'un groupe social mouvant et peu défini, celui des étudiants. Il mobilise pour cela une très large palette de sources, croisant des documents d'origine institutionnelle (divers fonds du RGANI et du RGASPI concernant l'action du PCUS dans le domaine de la politique visant les étudiants, certains fonds de l'organisation du Komsomol conservés au RGASPI, des fonds des cellules locales du PCUS au sein des facultés, qui se trouvent dans les archives municipales de Moscou, etc.) et des documents privés, tels que journaux 
intimes, mémoires, éléments de correspondance, entretiens. Les citations de la presse périodique étayent également l'analyse dans une très large mesure.

13 Cependant, prisonnière de catégories indigènes et d'un schéma explicatif binaire qui oppose résistance et conformité au pouvoir, l'auteur n'arrive pas à dépasser le niveau purement descriptif, laissant le lecteur sur sa faim quant aux mécanismes sociaux qui auraient conduit à la formation de tel ou tel foyer d'opinion. De plus, en construisant son raisonnement autour des événements politiques, l'auteur renonce d'emblée à ce que le titre de son ouvrage semblait promettre: étudier les étudiants en tant que milieu autonome, certes conditionné en partie par les instances de pouvoir, mais pas entièrement défini par elles.

\section{NOTES}

1. Cf. par exemple, E. Zubkova, Poslevoennoe obščestvo : politika i povsednevnost', 1945-1953, Moscou: ROSSPEN, 2000, ou N. B. Lebina et A. N. Čistikov, Obyvatel' i reformy : kartiny povsednevnoj žizni gorožan, Saint-Pétersbourg : Dmitrij Bulanin, 2003.

2. "Réactions des étudiants au début de la guerre froide ", "Critiques des étudiants vis-à-vis de la politique nationale ", «La personne de Stalin dans la conscience des étudiants et réactions à la mort du "chef" " (ch. III) ; "Lutte pour le pouvoir et réactions des étudiants soviétiques ", «Réactions des étudiants au rapport Hruščev intitulé "Le culte de la personnalité et ses conséquences" ", « Réactions des étudiants à la démission de Hruščev » (ch. IV). 